\title{
ZERO SKEW CLOCK ROUTING FOR FAST CLOCK TREE GENERATION
}

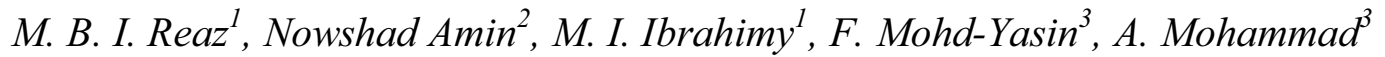 \\ ${ }^{1}$ Department of Electrical and Computer Engineering, International Islamic University Malaysia, 53100 \\ Kuala Lumpur, Malaysia \\ ${ }^{2}$ Dept. of Electrical, Electronic and System Engineering, Faculty of Engineering, National University of \\ Malaysia, 43600 UKM, Bangi, Selangor, Malaysia \\ ${ }^{3}$ Faculty of Engineering, Multimedia University, 63100 Cyberjaya, Selangor, Malaysia
}

\begin{abstract}
A Zero Skew Clock Routing Methodology has been developed to help design team speed up their clock tree generation process. The methodology works by breaking up the clock net into smaller partitions, then inserting clock buffers to drive each portion, and lastly, routing the connection from original clock source to each newly inserted clock buffers with zero skew. A few Perl scripts and a new Visual Basic based routing tool have been developed to support the methodology implementation. The routing algorithm used in this tool is based on the Exact Zero Skew Routing Algorithm. The methodology has been tested using a real design database and resulting in a significant improvement in the through put time required to complete the clock tree generation. This improvement is attributed to the ability to generate clock tree on much smaller portions of clock nets that supports of speeding up the clock tree generation process in IC design.
\end{abstract}

Index Terms- Zero skew, Clock routing, Clock tree generation, IC design

\section{INTRODUCTION}

At a deep submicron (DSM) silicon technology levels, it is now feasible to integrate all major function of an end product in a single system-on-a-chip (soc) silicon die [1]. However, almost all of these chips are still designed based on synchronous clock design. Physically, the clock signal is distributed from an external pad to all similarly clocked synchronizing elements through a distribution network that includes clock distribution logic and interconnects. It serves to unify the design by determining the precise instance in time that the synchronizing elements change state. In order to improve chip performance, i.e. to make the chip operates at faster clock speed; the chip designer must ensure that the clock signal is distributed to all the synchronizing elements in the design at the same time. Non-optimal clock behavior is typically caused by either one of these two phenomena: the unbalanced routing to the chip's synchronizing elements, or the non-symmetric behavior of the clock distribution CCECE/CCGEI May 5-7 2008 Niagara Falls. Canada $978-1-4244-1643-1 / 08 / \$ 25.00$ (C) 2008 IEEE logic [2]. The problem is further complicated by the massive size of modern chip design and the fact that the design usually utilizes more than one clock signal to sync up its function. Each of these clocks may operate at different frequencies [3]. therefore the common challenge faced by all chip designers is how can they create a perfect clock distribution scheme that could guarantee all the timing elements scattered all over the chip are activated by the same edge of the clock signal simultaneously. The maximum difference between the arrival times from the clock source to the elements is defined as the clock skew [4, 5]. The ideal case is to have all the elements sync up at the same time, which would mean no skew.

The benefits of a zero skew clock distribution are well known and have been proven to affect the performance of a chip significantly. Many researches have pointed out that minimizing clock skew is the most important task when designing a clock network. Works, such as [2, 6, 7], have led to many other studies and researches on how to balance a clock network and achieve the zero clock skew target. More recent studies, such as $[8,9]$ also reveal that reducing clock skew can help to minimize power consumption of large chips, which is very important for chips that are designed for low power application (longer battery life) such as mobile computers and cell phones.

\section{THE CLOCK TREE GENERATION PROBLEM AND THE PROPOSED SOLUTION}

As the chip size grows (in term of increasing number of functional elements), the clock nets get bigger as well. Clock nets have bigger fan-out, and have to be distributed over larger areas. It has been observed that existing clock tree generation (CTG) tool performance has deteriorated as the clock net fan out size grow bigger and bigger. The tool execution time is getting slower and slower, and it is taking more iteration before the user can get a result that matches the desired outcome. It is suspected that the tool may have hit the limit of its computation capability, and is not able to handle nets with large fan-out. Although the CTG tool has been used extensively and successfully before, this capability limitation is slowing the design process. 
Since the problem is the CTG tool capacity, the logical solution is to break the clock nets into smaller parts. This can be accomplished by partitioning the chip into several pseudo-partitions at the layout level, based on the cells placement, as opposed to partitioning at the RTL level, which will be a real design partitioning effort. Partitioning at RTL level will involve some changes to the chip architecture and would increase the complexity of this solution.

The pseudo-partitioning task will be executed after the completion of cells placement stage. The design engineer can output the placement information of the chip and analyze and decide the best way to partition each clock net. The engineer must also make sure that the size of each pseudo-partition should be well below the upper limit of what the CTG tool is capable of handling.

For each of the pseudo-partition, a new buffer will be inserted to act as a new clock source point for each pseudopartition. Each pseudo-partition will have its own clock source point, and that clock point will drive a smaller number of fan-out. The location of the clock source point will be greatly influenced by the floor plan of the chip and the placement location of the fan-out cells as well. Since the clock net is smaller, the CTG tool should perform well within expectation.

\section{REVIEWS ON CLOCK ROUTING ALGORITHMS}

There are many clock routing algorithms that have been proposed throughout the years. The aim of all of these algorithms is to deliver a zero or minimal skew clock routing and distribution.

One of the earliest clock routing algorithms is the HTree clock routing algorithm. While the H-Tree clock routing algorithm is widely used in the IC industry [10], their application is not suitable for IC design because cells placement in IC design is not symmetrical. This fact is also supported by the Power PC microprocessor design team. The team has conducted experiment on their chip and concluded that clock tree generation methodology with balance router ensure quick turnaround and produce more than $40 \%$ improvement over H-Trees [11].

The Method of Means and Median (MMM) algorithm has been proposed to overcome the H-Tree Algorithm shortcoming [2], but it also has its weaknesses. The difference in wire length from the clock source to each leaf cell can be as large as half the diameter of the chip. The algorithm effectiveness depends heavily on how the cut direction is decided, thus adding lethargy to the algorithm implementation.

The Recursive Geometric Matching (RGM) algorithm was proposed in 1991 [6]. It has been proposed to fix the unbalanced wire length problem seen in the MMM algorithm. This algorithm always yields clock tree with perfectly balance path length for trees of two, three, or four terminals, but does not necessarily minimize the skew as it did not consider the cell loading. Like the MMM algorithm, the algorithm cannot guarantee a balanced wire length on certain cases, and requires work around that reduces its effectiveness.

The Exact Zero Skew (EZS) algorithm was first proposed in 1991, by Dr Ren-Song Tsay, from IBM's (International Business Machine) T.J. Watson Research Center [7]. It considers delay balance instead of the wire length balance has been proposed to deliver better skew performance. The EZS algorithm adopts a bottom up process similar to that of the RGM algorithm. This algorithm achieves delay balance by elongating wires that have smaller delays, therefore, does not require any look ahead techniques that can slow down the execution.

In order to have a fair comparison, simulation exercise to test out the three different algorithms, namely, the MMM, the RGM, and the EZS algorithm, to route a clock net with 8 leaf cells has been performed. The wire used for routing was 1 micron wide and the wire resistance per unit length was $1 \mathrm{~m} \Omega$ and wire capacitance per unit length was $0.075 \mathrm{fF}$. Each unit length was equivalent to 1-micron meter. The die area was 8000 microns by 8000 microns. The overall comparison is summarized in the Table I.

The simulation results clearly show that the EZS algorithm gives the smallest skew $(0.004233 \mathrm{ps})$ and its amount is negligible. However, in this test case, the maximum path delay and the amount of wire length used to route the nets using EZS algorithm are only slightly lower compared to the other algorithm $(3.7 \%$ difference in maximum path delay difference and $0.4 \%$ in wire length). This is consistent with what is reported in [7]. Based on all these considerations, the EZS algorithm is recommended and proposed to be deployed in the implementation of the zero skew clock net routing tools.

Table I

SUMMARY OF THE COMPARISON

\begin{tabular}{|c|c|c|c|}
\hline Algorithm & $\begin{array}{c}\text { Max Path } \\
\text { Delay }(\mathrm{ps})\end{array}$ & Skew $(\mathrm{ps})$ & $\begin{array}{c}\text { Total Wire } \\
\text { Length }(\mu \mathrm{m})\end{array}$ \\
\hline MMM & 16.78469 & 1.883 & 88000 \\
\hline RGM & 16.21875 & 0.285 & 88000 \\
\hline EZS & 15.61857 & 0.004233 & 87610 \\
\hline
\end{tabular}

\section{IMPLEMENTATION OF THE SOLUTION}

The proposed solution is called Zero Skew Clock Routing (ZSCR) methodology. The methodology is presented in the flowchart shown in Fig. 1. It can actually be categorized in three stages. The first stage is the pseudo-partitioning stage. In this stage, the user analyzes the placement results and identifies how to pseudo-partition the clock networks. The user has to also determine the location of the new clock source for each pseudo-partition. The second stage is the clock trunk routing stage. For this stage, a software program based on the EZS algorithm had been created to perform the zero skew routing between the original clock sources to the 
new pseudo-partition clock sources. This part of the net is referred to as the clock trunk as it now became the main branch of the clock net. The third stage is the database update stage. In this stage, the results from stage 1 and stage 2 are integrated back into the database. This methodology is implemented in three stages.

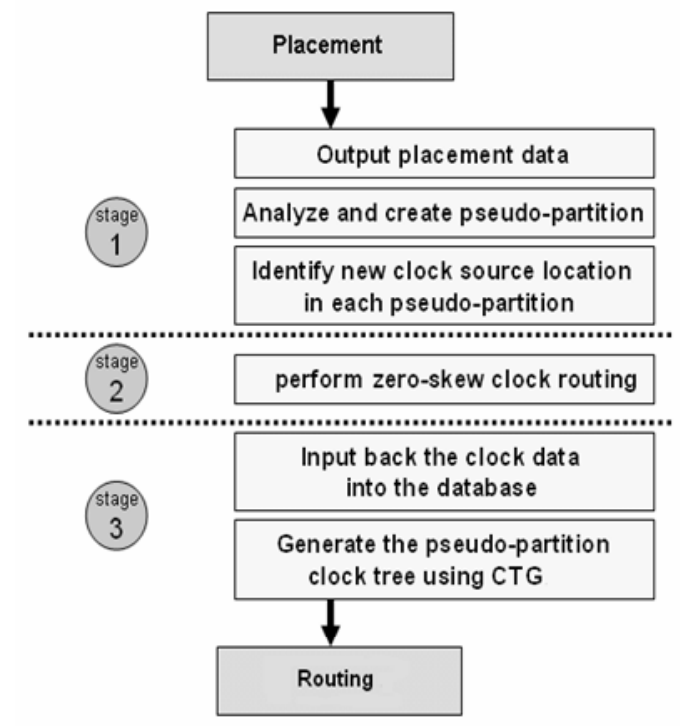

Fig. 1. Zero Skew Clock Routing Flowchart

\subsection{The Pseudo-partitioning Stage}

In the first stage, a couple of Perl scripts were written to help the user extract and analyze placement data as well as determine the pseudo-partition boundary and the location of the new clock source. A set of guidance on the decision making process of determining the pseudo-partition boundary and clock buffers location was given as well.

\subsection{The Clock Trunk Routing Stage}

For this stage, a software tool called Mojiii was created to perform the zero skew routing between the original clock sources to the new pseudo-partition clock sources. The routing algorithm used in this tool is based on the EZS algorithm that was authored by Dr Ren-Song Tsay, from IBM's (International Business Machine) T.J. Watson Research Center. The tool is written using Microsoft Visual Basic. The main input to this tool is the location of the original and pseudo-partition clock sources and the input pin capacitance of the pseudo-partition clock source. One of the outputs from the tool is a command file that instructs how to implement the routing in automatic place and routegraphical user interface (APR-GUI).

\subsection{Updating Database}

In the third and final stage, another set of Perl Scripts was coded to integrate back the results from stage 1 and stage 2 back into the database.

After completing all the three stages, the user continues with the normal clock tree generation process. Since the clock nets have been partitioned into smaller portions, the clock tree generation process completes faster and requires less iteration to meet the skew and delay specification.

\section{RESULTS AND DISCUSSION}

\subsection{Results of Calibration Test Cases}

To calibrate the tool accuracy and validate the legality of the methodology and its results, a couple of test cases have been conducted. The test cases use the gClk400m and sR32k nets. The nets are chosen because of their placement distribution reflect typical clock distribution on the chip. Net gClk400m has 2231 fan-out or leaf cells and net sR32k has 687 leaf cells. The number of leaf cells in this two test case is not very high, to allow faster evaluation, less tedious and more accurate result validation. The clock frequency for $\mathrm{gClk} 400 \mathrm{~m}$ is $400 \mathrm{MHz}$, the clock period is $2.5 \mathrm{~ns}$, and the maximum skews allowed are set to $5 \%$ of the clock period, which are 125 ps in this case. For the sR32k net, the clock frequency is $32 \mathrm{MHz}$, the clock period is $31.25 \mathrm{~ns}$, and the maximum skew allowed is also set to $5 \%$ of the clock period, which are 1562.5 ps in this case.

The net is routed in Mojiii to ensure zero skew routing from the clock source to the new buffers. The resistance per square length value is set to $0.001 \mathrm{~m} \Omega / \mu \mathrm{m}$ and the capacitance per length is set to $0.075 \mathrm{fF} / \mu \mathrm{m}$. These numbers are derived from the resistance per square value and the capacitance per square value of the routing layer, assuming that all the routing will be done with $1 \mu \mathrm{m}$ wire. The results of the routing are shown in the Fig. 2 for gClk400m net and in the Fig. 3 for sR32k net.

From the results calculated in Mojiii, the path delay for net $\mathrm{gClk} 400 \mathrm{~m}$, from the clock source to its leaf cells is 4.283 ps, with zero (negligible) skew and for net sR32k from the clock source to its leaf cells is $6.560 \mathrm{ps,} \mathrm{with} \mathrm{zero}$ (negligible) skew, as expected.

The routing for both nets is then implemented on the real database using two different routing layers. The results are shown in the Table II and III. 


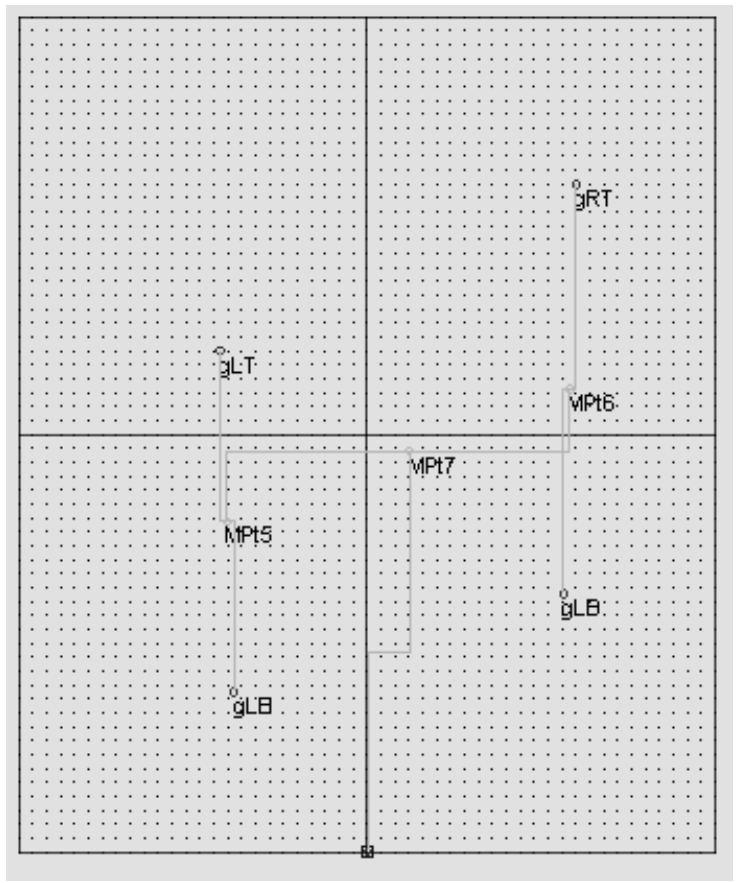

Fig. 2. Routing Results for Net gClk400m

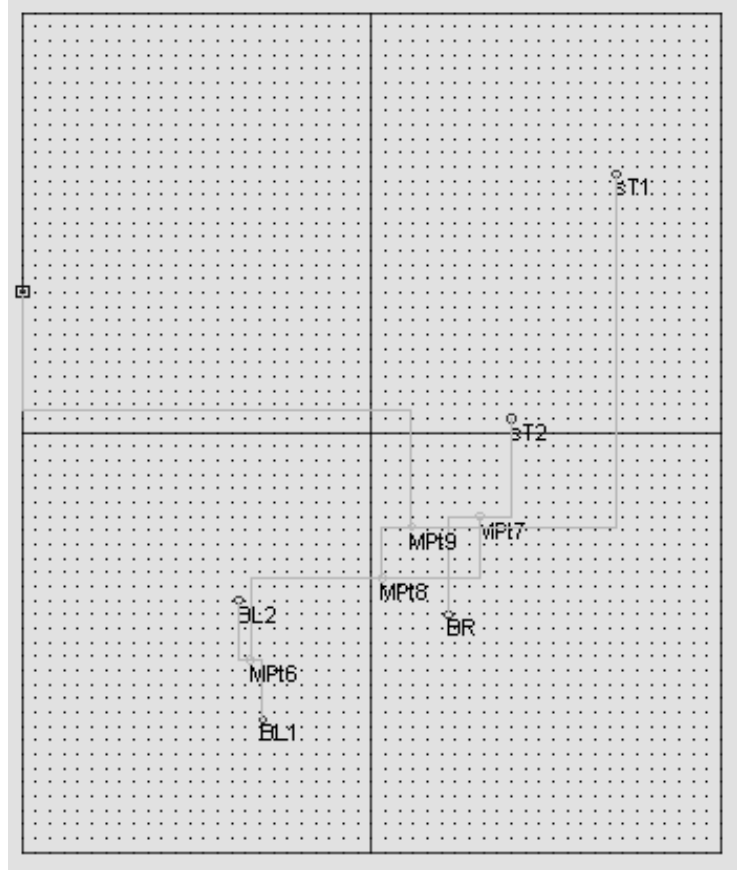

Fig. 3. Routing Results for Net sR32k

Another item that needs to be evaluated is the total time needed to generate the clock tree using this new methodology. The results are shown in Table IV. The throughput time is about 13 to $18 \%$ better for these test cases. However, it is believed that it will affect more significantly on larger clock nets.
Table II

EXTRACTED RESULTS FOR NET GCLK400M

\begin{tabular}{|l|l|l|l|l|}
\hline \multicolumn{1}{|c|}{ Cell } & \multicolumn{1}{|c|}{$\mathrm{X}$} & \multicolumn{1}{c|}{$\mathrm{Y}$} & $\begin{array}{c}\text { Input } \\
\text { Capacitance } \\
(\mathrm{fF})\end{array}$ & $\begin{array}{c}\text { Output to } \\
\text { input pin } \\
\text { path delay } \\
(\mathrm{ps})\end{array}$ \\
\hline gClk400m & 2504 & 3 & - & - \\
\hline gLB & 1545 & 1150 & 70 & 4.928844 \\
\hline gLT & 1445 & 3600 & 70 & 4.950264 \\
\hline gRB & 3910 & 1855 & 70 & 4.841353 \\
\hline gRT & 3998 & 4795 & 70 & 4.862765 \\
\hline Longest Delay & 4.950264 \\
\hline \multicolumn{2}{|l}{ Shortest Delay } & 4.841353 \\
\hline \multicolumn{2}{|l}{ Maximum Skew } & 0.108911 \\
\hline \multicolumn{2}{|l}{ Clock Period (400 MHz) } & 2500 \\
\hline \multicolumn{2}{|l}{ Percentage of skew over clock period } & $0.00436 \%$ \\
\hline
\end{tabular}

Table III

EXTRACTED RESULTS FOR NET SR32K

\begin{tabular}{|l|l|l|l|l|}
\hline Cell & \multicolumn{1}{|c|}{ X } & \multicolumn{1}{|c|}{ Y } & $\begin{array}{c}\text { Input } \\
\text { Capacitance } \\
(\mathrm{fF})\end{array}$ & $\begin{array}{c}\text { Output to } \\
\text { input pin } \\
\text { path delay } \\
(\mathrm{ps})\end{array}$ \\
\hline sR32k & 5 & 4006 & - & - \\
\hline BL1 & 1720 & 950 & 70 & 7.437194 \\
\hline BL2 & 1550 & 1800 & 70 & 7.474003 \\
\hline BR & 3050 & 1700 & 70 & 7.371831 \\
\hline ST2 & 3500 & 3100 & 70 & 7.338573 \\
\hline ST1 & 4250 & 4850 & 70 & 7.304272 \\
\hline Longest Delay & 7.470272 \\
\hline \multicolumn{2}{|l}{ Shortest Delay } \\
\hline \multicolumn{2}{|l}{ Maximum Skew } & 7.304272 \\
\hline \multicolumn{2}{|l}{ Clock Period (32 MHz) } & 0.1657316 \\
\hline
\end{tabular}

Table IV

THROUGHPUT-TIME COMPARISON

\begin{tabular}{|l|l|l|l|}
\hline \multicolumn{1}{|c|}{ Net } & $\begin{array}{c}\text { Original } \\
\text { (minutes) }\end{array}$ & $\begin{array}{c}\text { Mojiii } \\
\text { (minutes) }\end{array}$ & Improvement \\
\hline gClk400m & 261 & 226 & $13.4 \%$ \\
\hline sR32k & 145 & 119 & $17.93 \%$ \\
\hline
\end{tabular}

\subsection{Results of Evaluation on a Chip Database}

The ZSCR methodology was deployed on one of the chips. The chip was fabricated in 0.35 micron ( $\mathrm{L}_{\text {eff }}=0.25$ microns), $2.5 \mathrm{~V}$ CMOS technology with 6 metal layers. The chip die size was $7.2 \mathrm{~mm} \times 5.5 \mathrm{~mm}$ and contained 2.8 million transistors. The clock distribution network has to serve a large number of macro cells (18) along with approximately 32,000 master slave latch-pairs. The frequency of the chip's main clock is $250 \mathrm{MHz}$.

The main clock was first balanced using the original CTG tool. Then the balancing exercise was repeated using the ZSCR Methodology. The results from both run are compared in Table V. 
TABLE V

RESULTS COMPARISON

\begin{tabular}{|l|l|l|l|}
\hline \multicolumn{1}{|c|}{ Flow } & $\begin{array}{c}\text { Maximum } \\
\text { Path Delays } \\
(\mathrm{ns})\end{array}$ & $\begin{array}{c}\text { Maximum } \\
\text { Skew (ps) }\end{array}$ & $\begin{array}{c}\text { Throughput } \\
\text { Time } \\
(\text { days })\end{array}$ \\
\hline Original & 12.5621 & 180 & 14 days \\
\hline $\begin{array}{l}\text { Zero } \\
\text { Skew }\end{array}$ & 12.2873 & 174 & 7 days \\
\hline
\end{tabular}

Based on the results, the maximum path delays and maximum skews show some improvement, but are not significantly different. This is expected as the main underlying tools for balancing the clock net is still the same, which is the CTG tool. However, there is a significant $50 \%$ improvement in the through put time. This improvement is attributed to the ability to run CTG on much smaller portions of clock nets and to the ability to run it concurrently, i.e. running five smaller nets on five workstations in parallel.

The results of this evaluation validate that the ZSCR methodology has been correctly implemented and has produced valid results. It also confirms that the deployment of the methodology has achieved its goal of speeding up the clock tree generation process in IC design.

\section{CONCLUSION}

The ZSCR methodology has been shown to have helped the design team improve their overall throughput time when balancing the main clock network on the chip. There are still many areas of improvement that can be implemented.

The pseudo-partitioning stage can be further automated to eliminate the need for the user to manually analyze the placement. The efficiency of stage two of the methodology can be further improved to provide more accurate delay estimations and address other realistic layout concerns. One of the layout concerns is that the EZS algorithm does not strive for wire length minimization. Another area of improvement that should be implemented is wire-sizing.

\section{REFERENCES}

[1] P. Rashinkar, P. Paterson, L. Singh, "System-on-a-Chip Verification: Methodology and Techniques," New York: Kluwer Academic Publishers, 2001.

[2] M. A. B. Jackson, A. Srinivasan, E. S. Kuh, "Clock Routing for High-Performance ICs," Proceedings of the ACM/IEEE Design Automation Conference, pp.573-579, June 1990.

[3] M. C. Chi, S. H. Huang, "A Reliable Clock Tree Design Methodology for ASIC Designs," Chung Yuan Journal, Vol. 28, No. 3, pp. 115-122, 2000.
[4] T. Chao, Y. Hsu, J. Ho, "Zero Skew Clock Routing with Minimum Wirelength," IEEE Transaction on Circuits and Systems, Vol. 39 No. 11, pp. 799-814, 1992.

[5] Y. P. Chen, D. F. Wong, “An Algorithm for Zero Skew Clock Tree Routing with Buffer Insertion," Proceedings of the European Design and Test Conference, pp. 230-236, March 1996.

[6] A. Kahng, J. Cong, G. Robins, "High Performance Clock Routing Based on Recursive Geometric Matching," Proceeding of the ACM/IEEE Design Automation Conference, pp.322-327, June 1991.

[7] R. S. Tsay, "Exact Zero Skew," Proceedings of the IEEE International Conference on Computer Aided Design, pp.336-339, November 1991.

[8] S. Pullela, N. Menezes, L. T. Pillage, "Low Power IC Clock Tree Design," Proceedings of the Custom Integrated Circuits Conference, pp.263-266, May 1995.

[9] R. Y. Chen, N. Vijaykrishnan, M. J. Irwin, "Clock Power Issues in System-on-a-Chip Designs," Proceedings of the IEEE Computer Society Workshop on VLSI '99. pp. 48-53, April 1999.

[10] H. Bakoglu, J. T. Walker, J. D. Meindl, “A Symmetric Clock Distribution Tree and Optimized High Speed Interconnections for Reduced Clock Skew in ULSI and WSI Circuit," Proceedings of the IEEE International Conference on Computer Design, pp. 118122, October 1986.

[11] K. M. Carrig, A. M. Chu, F. D. Ferraiolo, J. G. Petrovick, P. A. Scott, and R.J. Weiss, "A clock Methodology for HighPerformance Microprocessors," Proceeding of the IEEE Custom Integrated Circuits Conference, pp. 119-122, May 1997. 
Intentional Blank Page

000028 